\title{
Gallbladder Kaposi Sarcoma
}

National Cancer Institute

\section{Source}

National Cancer Institute. Gallbladder Kaposi Sarcoma. NCI Thesaurus. Code C5842.

A Kaposi sarcoma arising from the gallbladder. 